\title{
Behavioral states in the fetus near term
}

H.D. Junge, $H$. Walter

On the basis of comparison and analogy it can be stated. that in the mature fetus behavioral states comparable to those seen in the newborn infant, i.e. quiet sleep, active sleep and even some kind of wakefulness do exist (1). These states can be identified fairly well from heart rate and motor activity patterns.

According to evaluation of 8 hour recordings of FHR and fetal movements in the fetus one complete sleep cycle of quiet sleep plus active sleep lasts about 75 $\mathrm{min}$, duration of one period of quiet sleep is $20 \mathrm{~min}$ and duration of one period of active sleep is nearly 1 hours.

Results of computer analysis of FHR macrofluctuation according to quantification methods developed by the author confirm state related differences of macroflucatuation pattern (2): Macrofluctuation amplitude for 1-min-segments of FHR is in the decile range from 3.0 to 7.6 BPM during quiet sleep and from 6.5 to 21.8 BPM during active sleep. Macrofluctuation frequency is in the decile range from 3.1 to 6.3 cycles per min during quiet sleep and from 2.1 to 4.9 cycles per min during active sleep.

Motor activity of the fetus is unevenly distributed in respect to quiet and active sleep: in $54 \%$ of all periods of quiet sleep no movements were felt by the mothers and in $80 \%$ of all periods of quiet sleep the total number did not exceed 2 movements. Contrary to that in all periods of active sleep except 1 the total number exceeded 2 movements. On the whole $90 \%$ of motor activity during sleep states occurs during active sleep. Because fetal movement are generally associated with accelerations these data refer to acceleration as well.

These data support the idea that spontaneous changes in centralnervous coordination or state behavior are the predominant causes for spontaneous and distinct changes in FHR and motor activity patterns in fetal wellbeing.

In a subsequent investigation fetal chest movement activity in respect to state behavior was evaluated. According to our results the influence of centralnervous coordination on chest movement activity is inconsistent: Half of the periods of quiet sleep were without any chest movements but in the other half chest movement activity ranged from 10 to $60 \%$ of state duration. Contrary to that in all periods of active sleep chest movement activity was present, ranging from 5 to $45 \%$ of state duration.

From our results it is obvious that for ante partum surveillance of the fetus non stress cardiotocography, fetal heart rate acceleration determination, fetal movement count and evaluation of fetal chest movement activity cannot be evaluated properly unless fetal state behavior is taken into account.

1. Junge, H.D.:

Behavioral states and state related heart rate and motor activity patterns in the newborn and the fetus ante partum - A comparative study.

I. Technique, illustration of recordings, and general results.

J. Perinat. Med. 7 (1979) 85

2. Junge, H.D.:

Behavioral states and state related heart rate and motor activity patterns in the newborn and the fetus ante partum - A comparative study.

II. Computer analysis of state related heart rate baseline and macrofluctuation pattern.

J. Perinat. Med. 7 (1979) 134

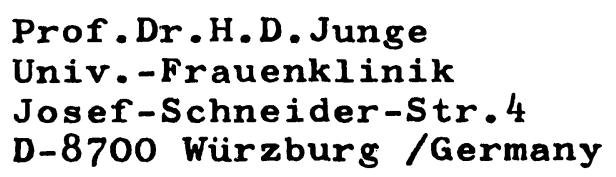

0300-5577/81/0091-0047\$2.00 Copyright by Walter de Gruyter \& Co. 